\title{
Burkholderia of Plant-Beneficial Group are Symbiotically Associated with Bordered Plant Bugs (Heteroptera: Pyrrhocoroidea: Largidae)
}

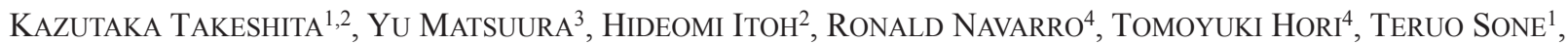 \\ Yoichi Kamagata ${ }^{1,2}$, Peter MergaerT ${ }^{1,2,5}$, and Yoshitomo KiKUCHI ${ }^{1,2 *}$ \\ ${ }^{1}$ Graduate School of Agriculture, Hokkaido University, Kita 9 Nishi 9, Kita-ku, Sapporo 060-8589, Japan; \\ ${ }^{2}$ Bioproduction Research Institute, National Institute of Advanced Industrial Science and Technology (AIST), Hokkaido Center, \\ 2-17-2-1 Tsukisamu-higashi, Toyohira-ku, Sapporo 062-8517, Japan; ${ }^{3}$ Graduate School of Environmental Science, Hokkaido \\ University, Kita 10 Nishi 5, Kita-ku, Sapporo, 060-0810, Japan; ${ }^{4}$ Environmental Management Research Institute, National Institute \\ of Advanced Industrial Science and Technology (AIST), 16-1 Onogawa, Tsukuba 305-8569, Japan; and Institute for Integrative \\ Biology of the Cell, Centre National de la Recherche Scientifique (CNRS), avenue de la Terrasse Bât. 34, 91198 Gif-sur-Yvette, France
}

(Received October 2, 2015-Accepted November 16, 2015-Published online December 9, 2015)

A number of phytophagous stinkbugs (order Heteroptera: infraorder Pentatomomorpha) harbor symbiotic bacteria in a specific midgut region composed of numerous crypts. Among the five superfamilies of the infraorder Pentatomomorpha, most members of the Coreoidea and Lygaeoidea are associated with a specific group of the genus Burkholderia, called the "stinkbugassociated beneficial and environmental (SBE)" group, which is not vertically transmitted, but acquired from the environment every host generation. A recent study reported that, in addition to these two stinkbug groups, the family Largidae of the superfamily Pyrrhocoroidea also possesses a Burkholderia symbiont. Despite this recent finding, the phylogenetic position and biological nature of Burkholderia associated with Largidae remains unclear. Based on the combined results of fluorescence in situ hybridization, cloning analysis, Illumina deep sequencing, and egg inspections by diagnostic PCR, we herein demonstrate that the largid species are consistently associated with the "plant-associated beneficial and environmental (PBE)" group of Burkholderia, which are phylogenetically distinct from the SBE group, and that they maintain symbiosis through the environmental acquisition of the bacteria. Since the superfamilies Coreoidea, Lygaeoidea, and Pyrrhocoroidea are monophyletic in the infraorder Pentatomomorpha, it is plausible that the symbiotic association with Burkholderia evolved at the common ancestor of the three superfamilies. However, the results of this study strongly suggest that a dynamic transition from the PBE to SBE group, or vice versa, occurred in the course of stinkbug evolution.

Key words: bordered plant bug, gut symbiosis, Burkholderia, evolution, symbiont replacement

A number of insects that exclusively feed on a nutritionallypoor diet or indigestible food such as plant sap, vertebrate blood, or woody materials, possess symbiotic microorganisms in their bodies $(5,6,38)$. In order to ensure the acquisition of beneficial microbes by their offspring, these insects have evolved sophisticated mechanisms for vertical symbiont transmission, such as ovarial transmission in aphids (50), milk-gland transmission in tsetse flies (3), and coprophagy in termites (29). Recent studies have revealed that several insects, including alydid bugs and allied heteropterans (37, 40), western flower thrips (14), and white flies (10), do not vertically transmit their symbiotic bacteria. Instead, these insects acquire symbiotic microorganisms from the environment every generation. Due to fragmented information for each insect lineage, the evolutionary history of symbiotic associations in species with horizontal transmission still remains unclear.

Among the Insecta, the infraorder Pentatomomorpha of the order Heteroptera may be regarded as an ideal model to elucidate the evolutionary history and mechanisms underlying symbiotic associations with horizontal transmission, since both, microbial symbioses with vertical and horizontal transmission, have evolved in this well-defined, single taxonomic

\footnotetext{
* Corresponding author. E-mail: y-kikuchi@aist.go.jp;
} Tel: +81-11-857-8909; Fax: +81-11-857-8980. group. The Pentatomomorpha, consisting of more than 12,500 species, is grouped into five superfamilies: Aradoidea, Pentatomoidea, Pyrrhocoroidea, Coreoidea, and Lygaeoidea $(25,62)$. Except for mycophagous Aradoidea, most members of Pentatomomorpha are phytophagous $(40,60)$. A number of phytophagous species develop crypts or tubular outgrowths in the midgut posterior region, wherein symbiotic bacteria are housed $(6,24,51)$. Members of the superfamily Pentatomoidea possess gammaproteobacterial symbionts and transmit microbes vertically through diverse mechanisms, such as egg smearing, capsule transmission, and jelly transmission $(1,22,26,27$, $32,33,39,42,55,56,67)$. On the other hand, most members of the superfamilies Coreoidea and Lygaeoidea are associated with symbionts of a specific clade of the betaproteobacterial Burkholderia, called the "stinkbug-associated beneficial and environmental (SBE)" group, which are maintained not by vertical transmission, but by environmental acquisition (23, $37,40,53)$. However, the partial vertical transmission of Burkholderia symbionts was recently reported in chinch bugs $(4,31)$.

In phylogenetic analyses based on morphological and molecular data $(25,28,73)$, the relationship between the pentatomomorphan superfamilies has been estimated as: $($ Aradoidea $+($ Pentatomoidea $+($ Pyrrhocoroidea $+($ Coreoidea + Lygaeoidea)))). On the basis of phylogeny, in conjunction with a broad survey of Burkholderia infections, we previ- 

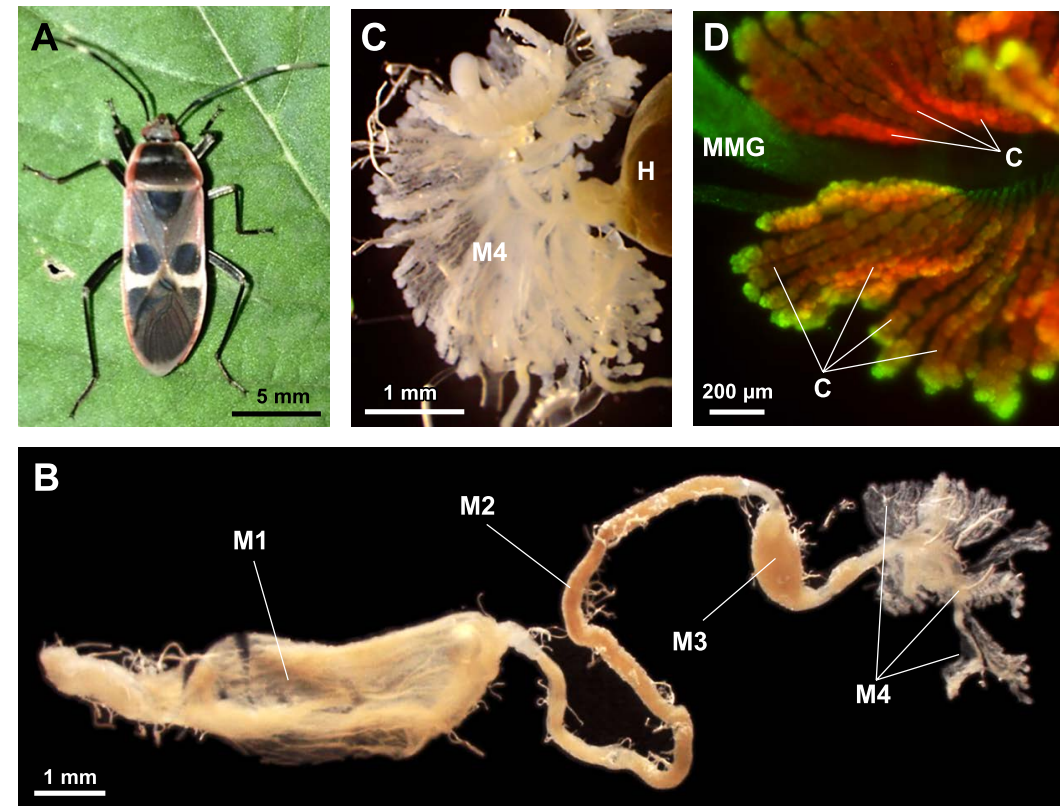

Fig. 1. The gut symbiotic organ in Physopelta gutta. (A) A female adult of $P$. gutta. (B) The dissected alimentary tract of a female adult of $P$. gutta. (C) An enlarged image of the midgut $4^{\text {th }}$ section (crypt-bearing symbiotic organ). (D) In vivo bacterial localization in the midgut $4^{\text {th }}$ section of $P$. gutta, visualized by whole-mount fluorescence in situ hybridization with a fluorochrome-labeled Betaproteobacteria-specific probe. Red signals indicate betaproteobacterial symbionts, whereas green signals visualize host insect nuclei stained with SYTOX Green. Abbreviations: M1, midgut $1^{\text {st }}$ section; M2, midgut $2^{\text {nd }}$ section; M3, midgut $3^{\text {rd }}$ section; M4, midgut $4^{\text {th }}$ section with tubular outgrowths; $\mathrm{H}$, hindgut; MMG, main duct of the midgut; $\mathrm{C}$, crypts.

ously hypothesized that the Burkholderia symbiosis evolved from a common ancestor of the superfamilies Coreoidea and Lygaeoidea (40). However, a recent extended survey by Sudakaran et al. (65) on the superfamily Pyrrhocoroidea revealed that several species of the pyrrhocoroid family Largidae, commonly known as bordered plant bugs, are associated with Burkholderia, which strongly suggests that the evolutionary origin of Burkholderia symbiosis is more ancient than we previously estimated (65). This finding suggests that Largidae is the key taxonomic group for elucidating the evolutionary history of Burkholderia symbiosis in the infraorder Pentatomomorpha. However, the phylogenetic position and biological nature of the Burkholderia symbionts in this family have not yet been investigated in detail.

In the present study, we characterized symbiotic associations in three largid species, Physopelta gutta (Fig. 1A), P. parviceps, and $P$. slanbuschii, with a focus on the phylogenetic placement of the symbionts. Our results demonstrated that bordered plant bugs closely associated with symbionts of the "plant-associated beneficial and environmental (PBE)" group of the genus Burkholderia, which is phylogenetically distinct from the SBE group, highlighting a dynamic transition from the PBE to SBE group, or vice versa, that occurred in the course of stinkbug evolution.

\section{Materials and Methods}

Insects

Insect samples used for cloning and sequencing were collected from different locations in Japan (Table 1) and preserved in acetone until used (21). In order to investigate Burkholderia infection in a natural insect population, 24 male and 24 female adults of $P$. gutta collected in Koshi, Kumamoto, Japan, were subjected to diagnostic PCR. Regarding egg inspections, 79 adults of $P$. gutta (38 males, 41 females) collected from the Koshi population were reared in plastic containers (diameter, $184 \mathrm{~mm}$; height, $120 \mathrm{~mm}$ ) at $25^{\circ} \mathrm{C}$ under a long-day regimen (16-h light, 8 -h dark), fed on sunflower seeds and distilled water containing $0.05 \%$ ascorbic acid, and 64 of the oviposited eggs were subsequently subjected to diagnostic PCR.

\section{DNA extraction}

Insects were dissected in phosphate-buffered saline (PBS) $\left(137 \mathrm{mM} \mathrm{NaCl}, 2.7 \mathrm{mM} \mathrm{KCl}, 8.1 \mathrm{mM} \mathrm{Na}_{2} \mathrm{HPO}_{4}, 1.5 \mathrm{mM} \mathrm{KH}_{2} \mathrm{PO}_{4}\right.$ [pH 7.4]) with fine forceps and micro-scissors under a dissection microscope, and the midgut fourth section, M4 (crypt-bearing symbiotic organ) (Fig. 1B), was isolated. Total DNA was extracted from the dissected symbiotic organ and eggs using the QIAamp DNA Mini Kit (QIAGEN, Hilden, Germany), according to the manufacturer's instructions. The quality of extracted DNA was confirmed by NanoDrop 1000 (Thermo Fisher Scientific, Waltham, MA, USA) and also by PCR amplification of the insect cytochrome oxidase I (COI) gene with the primers LCO1490 and HCO2198 (19) (see Table S1).

\section{Cloning and Sanger sequencing}

A $1.5-\mathrm{kb}$ fragment of the bacterial $16 \mathrm{~S}$ rRNA gene was amplified with the primers 16SA1 and 16SB1 (20) (Table S1). PCR amplification, the cloning of PCR products, and Sanger sequencing of the clones were performed as previously described $(36,48)$. The sequence reads were assembled with phredPhrap software $(17,18)$ followed by manual inspections.

\section{Molecular phylogenetic analyses}

Clone sequences were classified into operational taxonomic units (OTUs) by macqiime v1.6.0 (8) and Mothur v1.31.2 (61) based on the furthest-neighbor algorithm with $99 \%$ identity threshold. The sequences of clones were subjected to a BLASTN search using the BLAST program (7) against the nucleotide collection (nt) database (downloaded on Feb 2, 2015 from: ftp://ftp.ncbi.nlm.nih.gov/blast/ $\mathrm{db} /$ ). Multiple alignments were constructed by MAFFT v7.032b (L-INS-i) (35) and gap-including and ambiguous sites in the alignments were then removed. Phylogenetic relationships were recon- 
Table 1. Insect samples investigated in this study.

\begin{tabular}{|c|c|c|c|c|c|c|c|c|}
\hline \multirow{2}{*}{ Species } & \multirow{2}{*}{ Insect ID } & \multirow{2}{*}{ Instar } & \multirow{2}{*}{$\operatorname{Sex}^{\mathrm{a}}$} & \multirow{2}{*}{ Collection location (in Japan) } & \multirow{2}{*}{ Collection date } & \multirow{2}{*}{ Collector } & \multicolumn{2}{|c|}{ Accession No. } \\
\hline & & & & & & & Sanger sequencing & Deep sequencing \\
\hline \multicolumn{9}{|c|}{ Physopelta gutta } \\
\hline & Pgu1 & Adult & $\mathrm{F}$ & Iriomote Island, Okinawa & Jul 1, 2009 & T. Hosokawa & LC070051-LC070066 & DRR042177, DRZ007392 \\
\hline & Pgu2 & Adult & $\mathrm{F}$ & Koshi, Kumamoto & p 28, 2009 & Y. Kikuchi & LC070067-LC070079 & DRR042178, DRZ007393 \\
\hline & Pgu3 & Adult & $\mathrm{F}$ & Tsukuba, Ibaraki & ct 28,2009 & N. Kaiwa & LC070080-LC070091 & DRR042179, DRZ007394 \\
\hline & Pgu4 & 5 th & - & Tanoura, Kochi & Sep 16, 2009 & Y. Kikuchi & LC070092-LC070103 & DRR042180, DRZ007395 \\
\hline & Pgu5 & Adult & M & Nakamura, Kochi & May 24, 2003 & M. Takai & LC070104-LC070113 & DRR042181, DRZ007396 \\
\hline & Pgu6 & Adult & $\mathrm{F}$ & Amakusa, Kagoshima & Jun 26, 2009 & H. Toju & LC070114-LC070125 & DRR042182, DRZ007397 \\
\hline & Pgu7 & 5 th & - & Tanegashima Island, Kagoshima & Jul 30, 2009 & T. Hosokawa & LC070126-LC070134 & DRR042183, DRZ007398 \\
\hline & Pgu8 & Adult & M & Kirisl & 2008 & H. Тoju & LC070135-LC070144 & DRR042184, DRZ007399 \\
\hline & Pgu9 & Adult & $\mathrm{F}$ & Ishige & 2009 & T. Hosokawa & LC070145-LC070151 & DRR042185, DRZ007400 \\
\hline & Pgu10 & 5 th & - & Tsukuba, Ibaraki & & Y. Kikuchi & LC070152-LC070159 & DRR042186, DRZ007401 \\
\hline \multicolumn{9}{|c|}{ 20 } \\
\hline & Ppa1 & Adult & $\mathrm{F}$ & Atami, Shizuoka & Jul 12, 2003 & T. Hosokawa & LC070160-LC070170 & DRR042187, DRZ007402 \\
\hline & Рpa2 & Adult & M & da, Wakayama &, 2008 & T. Hosokawa & LC070171-LC070181 & DRR042188, DRZ007403 \\
\hline & Рpa3 & Adult & $\mathrm{F}$ & Tsuno & Jul 13, 2002 & M. Takai & LC070182-LC070193 & DRR042189, DRZ007404 \\
\hline \multirow{2}{*}{\multicolumn{9}{|c|}{ Lе }} \\
\hline & & & & & & & & \\
\hline & Psl1 & Adult & $\mathrm{F}$ & Ishigaki Island, Okinawa & Jul 14, 2002 & K. Kohno & LC070205-LC070214 & DRR042191, DRZ007406 \\
\hline
\end{tabular}

${ }^{a} \mathrm{~F}$, female; M, male; - , undetermined.

structed with MEGA 6 (69) under the Tamura-Nei $+\Gamma$ model of nucleotide substitution (68) based on the maximum likelihood (ML) and neighbor-joining (NJ) methods. The bootstrap values of 1,000 replicates for all internal branches were calculated.

\section{Whole-mount fluorescence in situ hybridization (wFISH)}

The dissected alimentary tracts of $P$. gutta were subjected to wFISH, as previously described $(31,45,49)$. In order to visualize symbiont localization in the midgut, an Alexa555-labeled, Betaproteobacteriaspecific oligonucleotide probe BET940 (15) (Table S1) was used. Host nuclei were visualized by SYTOX Green (Invitrogen, Carlsbad, CA, USA). The tissues were observed under a fluorescence microscope (DMI 4000 B, Leica, Wetzlar, Germany). A control assay with no probe was also performed in order to confirm probe-specific fluorescent signals.

\section{Diagnostic PCR}

The primer set 16SA1 and BurkPBE was used for the specific detection of the PBE group of Burkholderia (Table S1), targeting a $1.0-\mathrm{kb}$ fragment of the 16S rRNA gene. PCR amplification was performed with BIOTAQ DNA polymerase (BIOLINE, London, UK) and Ampdirect Plus buffer (Shimadzu, Kyoto, Japan) under the following temperature profile: an initial denaturation at $95^{\circ} \mathrm{C}$ for $10 \mathrm{~min}$, followed by 35 cycles of denaturation at $95^{\circ} \mathrm{C}$ for $30 \mathrm{~s}$, annealing at $52^{\circ} \mathrm{C}$ for $1 \mathrm{~min}$, and extension at $72^{\circ} \mathrm{C}$ for $1 \mathrm{~min}$. The specificity of the primer set was confirmed by checking the PCR amplification of the following target and non-target Burkholderia species/strains: target species were $B$. phymatum STM815 and $B$. phytofirmans PsJN (the PBE group of Burkholderia), and non-target species/strains were Burkholderia sp. strain RPE64, Burkholderia sp. strain RPE67, B. glumae BGR1, and B. thailandensis E264. Specificity was also confirmed by sequencing of the PCR product.

\section{Quantitative PCR ( $P$ PCR)}

DNA samples used for cloning and Sanger sequencing were subjected to qPCR of the bacterial $16 \mathrm{~S}$ rRNA gene in order to estimate the total number of bacteria colonizing the midgut crypts using a LightCycler 96 system (Roche Diagnostics, Basel, Switzerland). A 300-bp fragment of the bacterial 16S rRNA gene was amplified with the universal primer set 515F and 806R (9) (Table S1). The PCR reaction mixture, with a total volume of $20 \mu \mathrm{L}$, contained $2.0 \mu \mathrm{L}$ of 10× PCR buffer (Applied Biosystems, Foster City, CA, USA), $2.0 \mu \mathrm{L}$ of GeneAmp dNTP Mix (2 mM each of dATP, dTTP, dGTP, and dCTP; Applied Biosystems), $1.2 \mu \mathrm{L}$ of $25 \mathrm{mM} \mathrm{MgCl}_{2}$ solution (Applied Biosystems), $0.2 \mu \mathrm{L}$ of SYBR Green I (Molecular Probes,
Eugene, OR, USA), $0.4 \mu \mathrm{L}$ of the primer mixture solution $(5 \mu \mathrm{M}$ each of forward and reverse primers), $0.1 \mu \mathrm{L}$ of AmpliTaq Gold DNA polymerase (Applied Biosystems), 9.1 $\mu \mathrm{L}$ of distilled water, $1.0 \mu \mathrm{L}$ of dimethyl sulfoxide, and $4 \mu \mathrm{L}$ of extracted DNA. The PCR temperature profile was $95^{\circ} \mathrm{C}$ for $10 \mathrm{~min}, 45$ cycles of $95^{\circ} \mathrm{C}$ for $30 \mathrm{~s}$, $57^{\circ} \mathrm{C}$ for $30 \mathrm{~s}$ and $72^{\circ} \mathrm{C}$ for $30 \mathrm{~s}$. In order to calculate the absolute number of $16 \mathrm{~S}$ rRNA gene copies, 10-fold serial dilutions of the target PCR product of Burkholderia sp. SFA1 were also amplified, as described previously (31).

\section{Deep sequencing of the 16S rRNA gene}

DNA samples used for cloning, Sanger sequencing, and qPCR were subjected to deep sequencing of the 16S rRNA gene. By using a Miseq sequencer (Illumina, San Diego, CA, USA), amplicon sequencing of the V4 region of the bacterial 16S rRNA gene was performed with the universal primers 515F and 806R (9) (Table S1) and analyzed as previously described (31). After joining paired sequences and removing chimeric and low-quality sequences $(\mathrm{Q}$-score $<30)$, the resulting sequences were taxonomically assigned by RDP classifier ver. 2.10 .1 (72) with a 50\% confident threshold. In the OTU analysis of the Burkholderia community, Burkholderia sequences were retrieved and clustered into OTUs, which were defined as clusters having $<1 \%$ sequence differences, using the macqiime program (version 1.6.0) (8). Burkholderia clusters (i.e., $\mathrm{BCC} \& \mathrm{P}, \mathrm{PBE}$, and SBE) using the representative sequences of each OTU were identified by a BLASTN search against our collection of Burkholderia sequences. These clusters were used to reconstruct the phylogeny shown in Fig. 2.

\section{Nucleotide sequence accession numbers}

The nucleotide sequences of the 16S rRNA genes determined in this study have been deposited in the DDBJ/EMBL/GenBank nucleotide sequence database under the accession no. LC070051 to LC070214. The sequence reads of deep sequencing have been deposited in the DDBJ Sequenced Read Archive under the accession number DRA003821 (see also Table 1).

\section{Results}

Physopelta species form tubular crypts in the M4 section, which house numerous symbiotic bacteria

The midgut of $P$. gutta consisted of four morphologically distinct sections: the stomach-like $1^{\text {st }}$ section (M1), the tubu- 


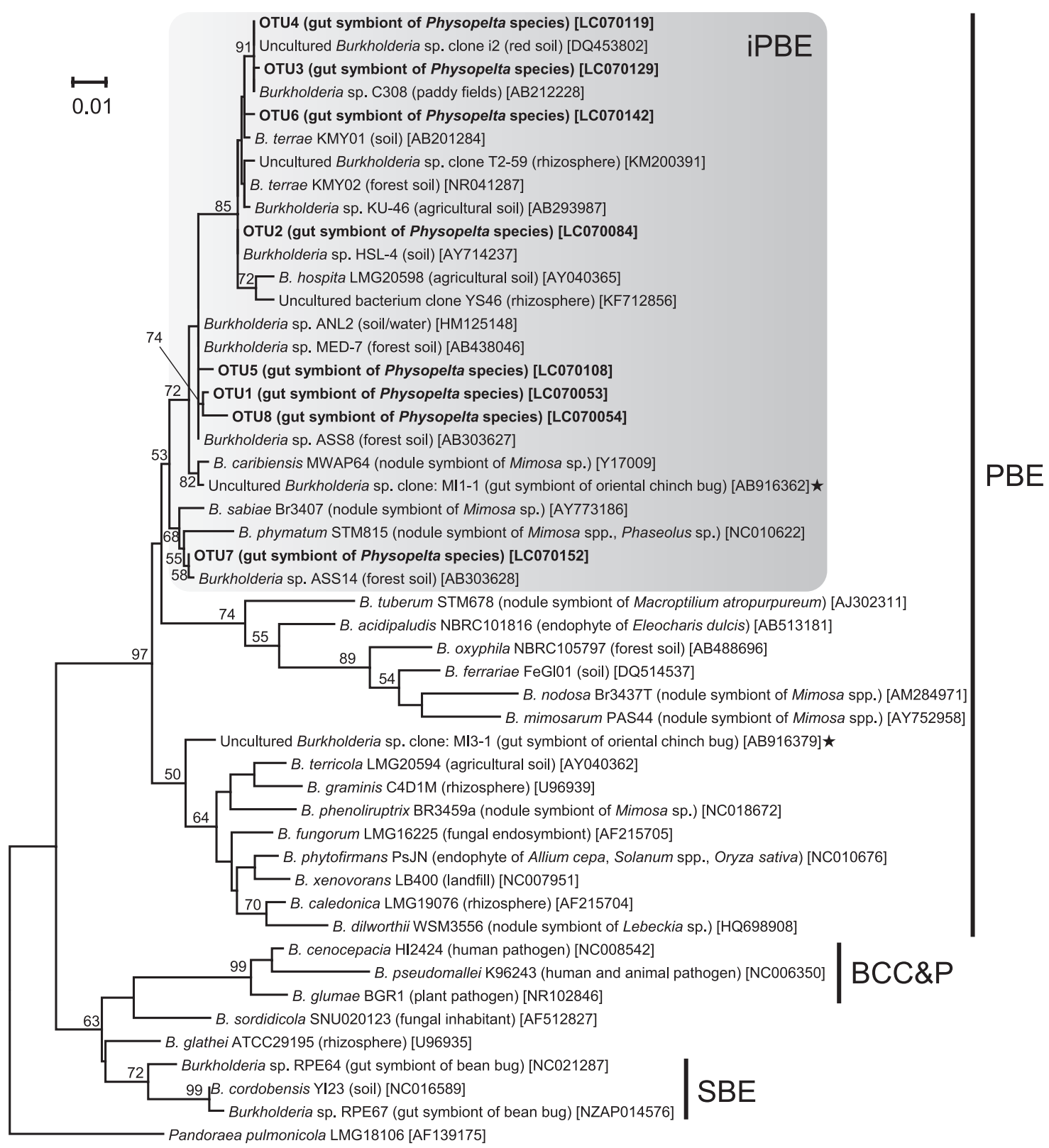

Fig. 2. Molecular phylogeny of the gut symbiotic bacteria of Physopelta species. The tree displays a maximum likelihood (ML) phylogeny of eight OTUs of the gut symbiotic bacteria identified from Physopelta gutta, P. parviceps and P. slanbuschii together with selected representatives of the different Burkholderia groups. The alignment of 1,356 nucleotide sites of the bacterial 16S rRNA gene was used. The gut symbionts of the Physopelta species are shown in bold. The origins or sources of isolation of the Burkholderia strains/sequences are represented in parentheses. Accession numbers in the DNA database (DDBJ/EMBL/GenBank) are shown in square brackets. Stars indicate gut symbionts detected from the oriental chinch bug Cavelerius saccharivorus (Lygaeoidea: Blissidae) in a previous study (31). The major Burkholderia clades (BCC\&P, SBE, and PBE) and the subclade "insect-associated PBE (iPBE)" are indicated on the right. Bootstrap values higher than 50\% are depicted at the nodes. A phylogeny of all of the 161 Burkholderia sequences obtained is shown in Fig. S1. ML and neighbor-joining (NJ) analyses gave essentially the same results (see Fig. S2).

lar $2^{\text {nd }}$ section (M2), the swollen $3^{\text {rd }}$ section (M3), and the crypt-bearing $4^{\text {th }}$ section (M4) (Fig. 1B). The midgut crypts appeared to be the tubular-outgrowth type and were arranged in two rows (Fig. 1C). The morphology and arrangement of crypts were similar to those in most members of Lygaeoidea (40), whereas the number of tubular outgrowths appeared to be larger in $P$. gutta. A similar midgut organization and M4 symbiotic organ were observed in $P$. parviceps and $P$. slanbuschii, and there was no sexual dimorphism. These morphological observations were identical to those reported in an early anatomical study by Miyamoto (51).
The dissected midguts of female adults of $P$. gutta, collected in Koshi, Kumamoto, Japan, were subjected to wFISH. Since the midgut microbes were identified as Burkholderia species (see below), the Betaproteobacteria-specific probe, BET940, targeting 16S rRNA was used to visualize the bacterial residents in the midgut. Bright signals were only detected in the M4 crypts, and not in the main duct of M4 (Fig. 1D). No signals were detected in the other parts of the midgut or in the tissues incubated with no probe (data not shown). This result confirmed that M4 is a symbiotic organ and that the bacteria are localized in its crypts. 
DNA samples extracted individually from the midgut crypts of 10 insects of $P$. gutta, four insects of $P$. parviceps, and one insect of $P$. slanbuschii, each representing a different Japanese population (Table 1), were subjected to qPCR in order to quantify the size of the bacterial population per insect. Quantitative PCR revealed that the numbers of copies of the $16 \mathrm{~S}$ rRNA gene were $8.9 \times 10^{6} \pm 1.0 \times 10^{7}$ (mean \pm standard deviation [SD], $n=10)$ in $P$. gutta, $1.2 \times 10^{7} \pm 6.9 \times 10^{6}($ mean \pm SD, $n=4)$ in $P$. parviceps, and $2.13 \times 10^{7}(n=1)$ in $P$. slanbuschii (Table 2 ). These values are similar to those reported for the symbiotic crypts of other stinkbug species (43), and indicate the very dense colonization of Physopelta midgut crypts.

Physopelta crypts are nearly exclusively colonized by Burkholderia

The 15 DNA samples from the midgut crypts of $P$. gutta,
$P$. parviceps, and $P$. slanbuschii, used in the qPCR analysis (Table 1) were subjected to the PCR amplification, cloning, and Sanger sequencing of a $1.5-\mathrm{kb}$ fragment of the bacterial $16 \mathrm{~S}$ rRNA gene. A total of 164 clones were sequenced and subjected to a BLAST search. The top BLAST hits of all sequences, except for three, were the 16S rRNA gene sequences of Burkholderia species. The three exceptional sequences exhibited the highest BLAST hits against either of two alphaproteobacterial species: Rickettsia bellii (accession number NR074484) and Lariskella arthropodarum (JQ726735). The 161 Burkholderia sequences were classified into eight OTUs based on the furthest-neighbor algorithm with a $99 \%$ sequence identity threshold (Table 3 ). The results obtained indicated that (i) all individuals were infected with multiple Burkholderia OTUs, except for one individual ( $P$. parviceps individual \#3 [sample ID, Ppa3]), which was solely infected

Table 2. Quantitative PCR and deep sequencing of $16 \mathrm{~S}$ rRNA genes of symbiotic microbiota in midgut crypts.

\begin{tabular}{|c|c|c|c|c|c|c|c|c|}
\hline \multirow[b]{2}{*}{ Insect ID } & \multirow[b]{2}{*}{ No. of copies ${ }^{\mathrm{a}}$} & \multicolumn{7}{|c|}{ No. of sequences ${ }^{b}$} \\
\hline & & Bacteria Total & $\begin{array}{l}\text { Burkholderia } \\
\text { Total }^{\mathrm{c}}\end{array}$ & $\begin{array}{c}\text { Burkholderia } \\
\text { Total }^{\mathrm{d}}\end{array}$ & $\mathrm{PBE}^{\mathrm{d}}$ & $\mathrm{BCC} \& \mathrm{P}^{\mathrm{d}}$ & $\mathrm{SBE}^{\mathrm{d}}$ & $\mathrm{UNC}^{\mathrm{d}, \mathrm{e}}$ \\
\hline \multicolumn{9}{|l|}{ Physopelta gutta } \\
\hline Pgu1 & $1.53 \times 10^{7}$ & 26,692 & 26,339 & $21,644(7)$ & $21,417(3)$ & 0 & $168(2)$ & $59(2)$ \\
\hline Pgu2 & $5.94 \times 10^{6}$ & 30,945 & 30,828 & $28,104(4)$ & $27,949(2)$ & 0 & $155(2)$ & 0 \\
\hline Pgu3 & $3.30 \times 10^{7}$ & 32,864 & 32,830 & $28,672(3)$ & $28,404(1)$ & 0 & $268(2)$ & 0 \\
\hline Pgu4 & $3.27 \times 10^{6}$ & 25,564 & 25,554 & $21,518(3)$ & $21,416(1)$ & 0 & $79(1)$ & $23(1)$ \\
\hline Pgu5 & $5.12 \times 10^{5}$ & 19,288 & 19,020 & $16,513(2)$ & $16,474(1)$ & 0 & 39 (1) & 0 \\
\hline Pgu6 & $1.23 \times 10^{7}$ & 26,653 & 26,588 & $24,200(4)$ & $24,000(1)$ & $31(1)$ & $169(2)$ & 0 \\
\hline Pgu 7 & $1.60 \times 10^{6}$ & 26,452 & 26,420 & $24,604(4)$ & 24,299 (1) & 0 & $276(2)$ & $29(1)$ \\
\hline Pgu8 & $1.53 \times 10^{6}$ & 51,568 & 50,697 & $46,290(3)$ & $45,946(1)$ & 0 & $344(2)$ & 0 \\
\hline Pgu9 & $1.34 \times 10^{7}$ & 16,660 & 16,540 & $12,331(5)$ & $12,211(1)$ & 0 & $98(3)$ & $22(1)$ \\
\hline Pgu10 & $2.02 \times 10^{6}$ & 72,410 & 72,348 & $66,471(4)$ & $66,068(3)$ & 0 & $403(1)$ & 0 \\
\hline \multicolumn{9}{|l|}{ P. parviceps } \\
\hline Ppal & $4.28 \times 10^{6}$ & 28,933 & 28,727 & $26,534(4)$ & $26,296(1)$ & 0 & $238(3)$ & 0 \\
\hline Ppa2 & $8.21 \times 10^{6}$ & 87,816 & 83,302 & $70,236(6)$ & $69,326(1)$ & 149 (1) & $680(3)$ & $81(1)$ \\
\hline Рpa3 & $1.72 \times 10^{7}$ & 21,337 & 21,070 & $19,494(6)$ & $19,226(1)$ & $29(1)$ & $215(3)$ & $24(1)$ \\
\hline Ppa4 & $1.83 \times 10^{7}$ & 21,153 & 21,051 & $18,835(3)$ & $18,741(1)$ & 0 & $74(1)$ & $20(1)$ \\
\hline \multicolumn{9}{|l|}{ P. slanbuschii } \\
\hline Psl1 & $2.13 \times 10^{7}$ & 14,794 & 14,623 & $12,997(3)$ & $12,897(1)$ & 0 & $100(2)$ & 0 \\
\hline
\end{tabular}

a The number of $16 \mathrm{~S}$ rRNA gene copies per individual was determined by qPCR.

$\mathrm{b}$ The numbers in parentheses indicate the number of OTUs.

${ }^{c}$ Assignment was performed by the RDP classifier with a 50\% confidence threshold.

$\mathrm{d}$ The indicated number is only for reproducible OTUs containing $>0.1 \%$ Burkholderia sequences in each library.

${ }^{\mathrm{e}}$ The number of sequences assigned as unclassified Burkholderia species.

Table 3. Numbers of $16 \mathrm{~S}$ rRNA gene clones assigned in each OTU of the Burkholderia.

\begin{tabular}{|c|c|c|c|c|c|c|c|c|c|}
\hline \multirow{2}{*}{ Insect ID } & \multicolumn{8}{|c|}{ Burkholderia } & \multirow{2}{*}{ Insect total } \\
\hline & OTU1 & OTU2 & OTU3 & OTU4 & OTU5 & OTU6 & OTU7 & OTU8 & \\
\hline \multicolumn{10}{|l|}{ Physopelta gutta } \\
\hline Pgul & 5 & 1 & 4 & 2 & & & 3 & 1 & 16 \\
\hline Pgu2 & 10 & 3 & & & & & & & 13 \\
\hline Pgu3 & 4 & 6 & 1 & & 1 & & & & 12 \\
\hline Pgu4 & 1 & 5 & 4 & & 2 & & & & 12 \\
\hline Pgu5 & 3 & & & & 6 & & & & 9 \\
\hline Pgu6 & & 4 & 3 & 5 & & & & & 12 \\
\hline Pgu7 & 2 & & 5 & 2 & & & & & 9 \\
\hline Pgu8 & 3 & 2 & & & 1 & 4 & & & 10 \\
\hline Pgu9 & & 3 & 2 & 2 & & & & & 7 \\
\hline Pgu10 & 1 & 1 & 1 & & & & 5 & & 8 \\
\hline \multicolumn{10}{|l|}{$P$. parviceps } \\
\hline Ppa1 & 3 & 4 & & 4 & & & & & 11 \\
\hline Ppa2 & 8 & & & & 1 & & & & 9 \\
\hline Ppa3 & 12 & & & & & & & & 12 \\
\hline Рpa4 & 1 & 10 & & & & & & & 11 \\
\hline \multicolumn{10}{|l|}{ P. slanbuschii } \\
\hline Psl1 & & & 3 & 1 & & 6 & & & 10 \\
\hline OTU total & 53 & 39 & 23 & 16 & 11 & 10 & 8 & 1 & 161 \\
\hline
\end{tabular}


with Burkholderia OTU1, (ii) most of the OTUs were shared by two or three Physopelta species, and (iii) OTU1 was the most frequently detected, at 53 out of 161 clones (32.9\%); however, OTU1 was not identified in P. slanbuschii (Table 3).

\section{Phylogenetic placement of Burkholderia symbionts among} plant-associated Burkholderia

The genus Burkholderia, which is a metabolically and ecologically diverse group in the Betaproteobacteria, is grouped into three phylogenetically and ecologically distinct clades: the first clade consists of a large number of human, animal, and plant pathogens, including B. cepacia, B. pseudomallei, $B$. mallei, and their allied species, designated as the " $B$. cepacia complex and B. pseudomallei (BCC\&P)" group $(13,59,63)$; the second clade includes a number of plant growth-promoting rhizobacteria and nodule-forming plant symbionts, assigned as the PBE group (63); the third clade mainly consists of gut symbionts of the Coreoidea and Lygaeoidea stinkbugs, assigned as the $\operatorname{SBE}$ group $(31,41)$.

The ML tree of Burkholderia OTUs detected from the midgut crypts of the Physopelta species is shown in Fig. 2 (see also Fig. S1 showing the phylogeny of all 161 Burkholderia sequences identified in this study). All Burkholderia detected in the symbiotic organ of Physopelta species were placed into the PBE group, in which Physopelta-associated Burkholderia formed a group with the rhizosphere species $B$. hospita and $B$. terrae, and the nodule-forming legume symbionts $B$. caribensis, B. phymatum, and B. sabiae (Fig. 2). This PBE subclade, tentatively named here as the "insect- and plant-associated beneficial and environmental (iPBE)" group, was supported by a $53 \%$ bootstrap value in the ML analysis. In this subclade, Physopelta-associated Burkholderia did not form a monophyletic group; instead, they clustered within the group of the rhizosphere species and nodule-forming legume symbionts (Fig. 2). The NJ phylogenetic tree showed a similar result, wherein all Burkholderia OTUs detected from the Physopelta species belonged to the iPBE clade with a $75 \%$ bootstrap value (Fig. S2).

The 15 insect samples used for qPCR and cloning analyses were subjected to Illumina deep sequencing of the 16S rRNA genes, resulting in 14,794 to 87,816 reads with a median of 26,653 reads for each sample (Table 2). In all 15 insects of the three Physopelta species, Burkholderia dominantly (>94\%) occupied bacterial communities in the midgut crypts (Fig. 3A and Table 2). Further analyses demonstrated that the dominant Burkholderia belonged to the PBE group (Fig. 3B and Table 2). These results were consistent with those of the cloning and Sanger sequence analyses. However, since only a 250-bp fragment of the 16S rRNA gene was analyzed by deep sequencing, detailed phylogenetic placements of Burkholderia, such as clustering of the iPBE group, were not possible.

In order to further confirm the prevalence of PBE Burkholderia in natural populations of the Physopelta species, a larger collection of 48 adult individuals of $P$. gutta (24 males and 24 females), collected from Koshi, Kumamoto, Japan, was subjected to the specific PCR detection of the PBE group of Burkholderia. All of the 48 insects were positive for infection, indicating the high prevalence of the PBE group of Burkholderia in natural populations of Physopelta insects.

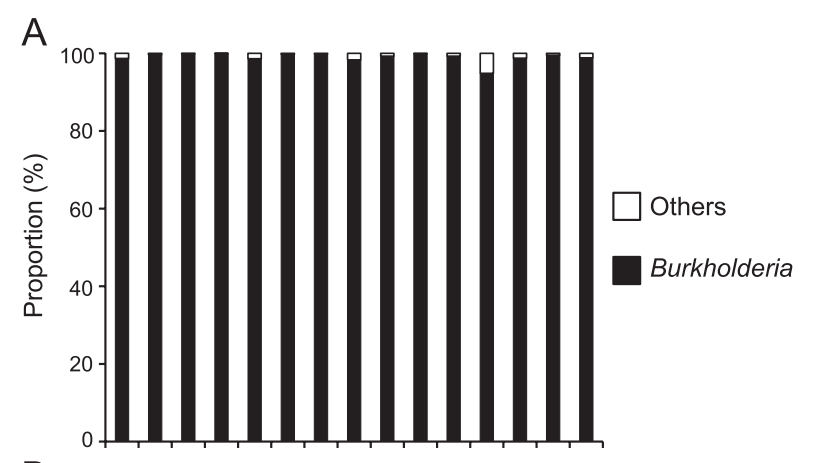

B

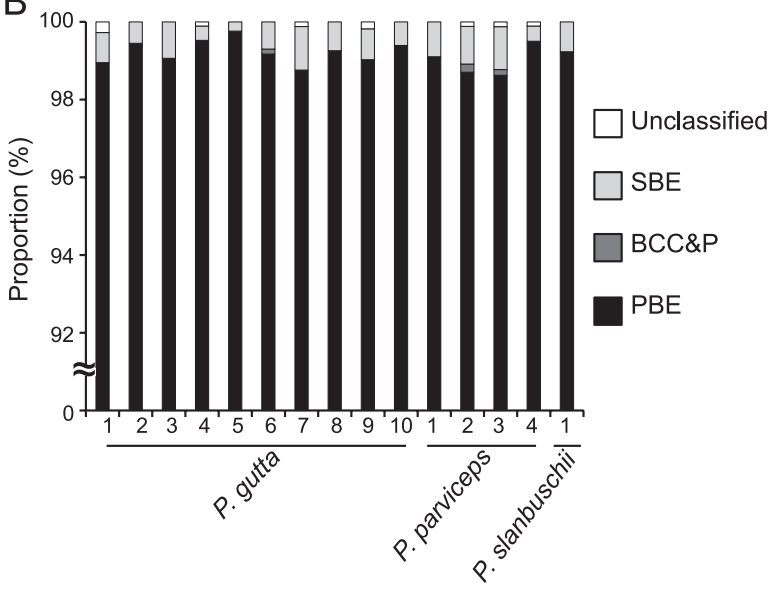

Fig. 3. Taxonomic composition of symbiotic microbiota in midgut crypts of Physopelta species. (A) Genus level composition. (B) Burkholderia clade level composition. The Illumina deep sequences annotated as Burkholderia were determined by using a BLASTN search against the reference sequences used in Fig. 2. On the basis of $>99 \%$ sequence identity, these sequences were categorized into any of the three major Burkholderia groups (BCC\&P, SBE, or PBE). Note that in all of the individuals of the three Physopelta species, sequences of the PBE group of Burkholderia accounted for $>93 \%$ of the total bacterial sequences (see Table 2 for more detailed information).

\section{Possible horizontal transmission of Burkholderia symbionts}

In order to clarify the transmission mode of the symbionts, we inspected 64 eggs of $P$. gutta by diagnostic PCR with the PBE-specific primer set. A faint band migrating at the expected size was only detected in two egg samples, while all others were negative (the detection rate was 3.1\%: positive/ total investigated $=2 / 64$ ). This result strongly suggests that symbionts are rarely transmitted vertically, but are essentially acquired horizontally.

\section{Discussion}

Based on the results of the present study, we conclude that: (i) Physopelta species possess a dense population of $10^{5}$ to $10^{7}$ cells per insect of symbiotic bacteria in the tubular-type midgut crypts; (ii) symbiotic bacteria belong to the PBE group of the genus Burkholderia, wherein the symbionts belong to a specific clade, the iPBE, which includes plant rhizosphere Burkholderia species and nodule-forming Burkholderia species; (iii) in this clade, Physopelta-associated, soil-isolated, rhizosphere and nodule-forming strains do not form coherent groups, but are intermixed; (iv) insects frequently harbor multiple strains/species of Burkholderia symbionts, repre- 
senting host specificity for iPBE species, but a relaxed specificity at the strain level; (v) eggs are rarely infected with Burkholderia symbionts, indicating infrequent vertical transmission; and, thus, it is plausible that (vi) largid bugs do not transmit Burkholderia symbionts vertically, but acquire them from the ambient environment every generation, as reported for Coreoidea and Lygaeoidea $(37,40)$. The gut microbiota of other largid species belonging to the genus Largus, which was sequenced previously (65), were also dominated by the PBE group of Burkholderia (data not shown), suggesting that the Largidae family is consistently associated with this group of Burkholderia.

In the infraorder Pentatomomorpha, the superfamilies Pyrrhocoroidea, Coreoidea, and Lygaeoidea form a monophyletic group $(25,28,73)$. The Largidae of Pyrrhocoroidea (this work; 65) as well as most members of Coreoidea and Lygaeoidea $(23,37,40,53)$ are associated with environmentallyacquired Burkholderia, while members of mycophagous Aradoidea lack gut symbionts (40) and those of Pentatomoidea harbor vertically-transmitted Gammmaproteobacteria $(1,22$, $26,27,32,33,39,42,55,56,67)$ (Fig. S3). As recently proposed by Sudakaran et al. (65), these results strongly suggest that the symbiotic association with environmentally-acquired Burkholderia evolved at a common ancestor of the superfamilies Pyrrhocoroidea, Coreoidea, and Lygaeoidea (Fig. S3), and that the midgut crypts as well as Burkholderia symbionts were lost in several families including the pyrrhocoroid family Pyrrhocoridae, which is the sister group of Largidae and harbors diverse Actinobacteria and Firmicutes symbionts in the M3 midgut region $(34,46,47,57,64)$. Although this hypothesis is plausible, the evolutionary history of Burkholderia symbiosis in Pentatomomorpha may be more complex than originally expected. We previously reported that Coreoidea and Lygaeoidea are consistently associated with the SBE group of Burkholderia (23, 37, 40, 53) (see also Fig. 2 and S3). On the other hand, the present study revealed that the largid species are closely associated with the PBE group of Burkholderia, which is phylogenetically distinct from the SBE group (Fig. 2), indicating that a dynamic transition from the PBE to SBE group of Burkholderia, or vice versa, occurred during the course of Pentatomomorpha evolution (Fig. S3).

At this stage, the transition history of Burkholderia symbionts remains unclear, and there are several evolutionary hypotheses. The high prevalence of the SBE group of Burkholderia in two out of the three superfamilies supports an evolutionary scenario in which stinkbugs were initially associated with the SBE group of Burkholderia and shifted to the PBE group of Burkholderia in the Largidae lineage (Fig. S3). Alternatively, on the grounds that (i) the Pyrrhocoroidea superfamily is phylogenetically placed in the basal branch of the three Burkholderia-associated superfamilies (Fig. S3) $(28,73)$, (ii) members of the family Largidae and superfamily Lygaeoidea commonly develop tubular-type midgut crypts, and (iii) in addition to the largid species, some species of Lygaeoidea are partially associated with the PBE group of Burkholderia (Fig. 2) $(4,31)$, it is possible that stinkbugs were initially associated with the PBE group of Burkholderia and were then replaced by the SBE group of Burkholderia in the common ancestors of the Coreoidea and Lygaeoidea superfamilies. In addition, it should be also taken into account that both the PBE- and the SBE-group Burkholderia were associated with the common ancestor and have been specialized in each stinkbug lineage. Further broad surveys and phylogenetic studies of hosts and symbionts are still needed.

The cohesive phylogenetic pattern of Physopelta-associated and plant-associated Burkholderia (Fig. 2) implies that members of the iPBE clade have a unique double lifestyle; these Burkholderia may be frequently transmitted between insects and plants, and symbiotically associated with both organisms or infect both hosts from the proximate environment. iPBE Burkholderia, although speculative, may have adaptations that make them well-suited for symbiotic life with these two very different types of hosts. Lifestyle transitions between these hosts may be possible by the horizontal gene transfer of gene cassettes carrying symbiotic functions specific for one or the other host, e.g., nodulation and nitrogen fixation genes for symbiosis with legumes. Since the Physopelta species investigated in the present study mainly feed on Mallotus trees (Malpighiales: Euphorbiaceae) in natural fields $(2,30)$, it is of great interest to inspect the community compositions of Burkholderia and their biological functions in Mallotus trees, which will lead to a more comprehensive understanding of the lifestyle of the iPBE group of Burkholderia.

Previous studies reported that the elimination of symbiotic bacteria in diverse stinkbug species resulted in retarded growth, reduced body size, high mortality, reduced fecundity, and/or abnormal body coloration in host insects $(4,26,33,37$, $42,43,57,58,67)$, indicating that symbiotic bacteria play a pivotal metabolic role in stinkbug hosts. In the Physopelta species, the well-developed symbiotic organ (Fig. 1), high symbiont density in the midgut crypts (Table 2), and high prevalence of symbionts in the host population strongly suggest an intimate host-symbiont interaction. In the iPBE group, $B$. caribensis, B. phymatum, and B. sabiae are known as nodule-forming symbionts in Mimosa species, wherein the symbionts fix atmospheric dinitrogen and provide the host with organic nitrogen compounds $(11,12,16,70)$. Many members of the PBE group of Burkholderia are recognized as plant growth-promoting rhizobacteria (PGPR) that consume ethylene by ACC (1-aminocyclopropane-1-carboxylic acid) deaminase and promote root elongation $(54,63,66)$. Some of the other members of the PBE group are known for their defensive abilities against phytopathogenic fungi $(63,71)$. These biological abilities may be involved in the symbiotic association with largid insects. The environmentally transmitted nature of largid symbionts implies their cultivability on axenic media; however, we have not yet succeeded in isolating symbionts from the midgut crypts. As demonstrated previously in the bean bug Riptortus pedestris $(44,52)$, cultured symbiotic Burkholderia will provide a unique opportunity not only to clarify the biological functions of iPBE symbionts in largid bugs, but also to comprehensively understand the mechanisms responsible for stabilizing stinkbug-Burkholderia symbiotic associations.

\section{Acknowledgements}

We thank T. Hosokawa, N. Kaiwa, M. Takai, H. Toju, and K. Kohno for collecting and providing insect samples, N. Nakamura for technical assistance, and M. Terashima for helpful comments. This 
study was supported by the Ministry of Education, Culture, Sports, Science and Technology (MEXT) KAKENHI (grant number 26117732 and 15H05638) to Y.K., and the Japan Society for the Promotion of Science (JSPS) Invitation Fellowships for Research in Japan (grant number L14556) to P.M.

\section{References}

1. Abe, Y., K. Mishiro, and M. Takahashi. 1995. Symbiont of brownwinged green bug, Plautia stali SCOTT. Jpn. J. Appl. Entomol. Zool. 39:109-115.

2. Ahmad, I., and C.W. Schaefer. 1987. Food plant and feeding biology of the Pyrrhocoroidea (Hemiptera). Phytophaga 1:75-92.

3. Attardo, G.M., C. Lohs, A. Heddi, U.H. Alam, S. Yildirim, and S. Aksoy. 2008. Analysis of milk gland structure and function in Glossina morsitans: Milk protein production, symbiont populations and fecundity. J. Insect Physiol. 54:1236-1242.

4. Boucias, D.G., A. Garcia-Maruniak, R. Cherry, H. Lu, J.E. Maruniak, and V.U. Lietze. 2012. Detection and characterization of bacterial symbionts in the Heteropteran, Blissus insularis. FEMS Microbiol. Ecol. 82:629-641.

5. Bourtzis, K., and T.A. Miller. 2003. Insect Symbiosis. CRC Press, Boca Raton, FL.

6. Buchner, P. 1965. Endosymbiosis of Animals with Plant Microorganisms. Interscience Publishers, New York.

7. Camacho, C., G. Coulouris, V. Avagyan, N. Ma, J. Papadopoulos, K. Bealer, and T.L. Madden. 2009. BLAST+: architecture and applications. BMC Bioinformatics 10:421.

8. Caporaso, J.G., J. Kuczynski, J. Stombaugh, et al. 2010. QIIME allows analysis of high-throughput community sequencing data. Nat. Methods 7:335-336.

9. Caporaso, J.G., C.L. Lauber, W.A. Walters, et al. 2012. Ultra-highthroughput microbial community analysis on the Illumina HiSeq and MiSeq platforms. ISME J. 6:1621-1624.

10. Caspi-Fluger, A., M. Inbar, N. Mozes-Daube, N. Katzir, V. Portnoy, E. Belausov, M.S. Hunter, and E. Zchori-Fein. 2012. Horizontal transmission of the insect symbiont Rickettsia is plant-mediated. Proc. Biol. Sci. 279:1791-1796.

11. Chen, W.-M., S.M. de Faria, R. Straliotto, et al. 2005. Proof that Burkholderia strains form effective symbioses with legumes: a study of novel Mimosa-nodulating strains from South America. Appl. Environ. Microbiol. 71:7461-71.

12. Chen, W.-M., S.M. de Farja, J.H. Chou, E.K. James, G.N. Elliott, J.I. Sprent, C. Bontemps, J.P.W. Young, and P. Vandamme. 2008. Burkholderia sabiae sp. nov., isolated from root nodules of Mimosa caesalpiniifolia. Int. J. Syst. Evol. Microbiol. 58:2174-2179.

13. Coenye, T., and P. Vandamme. 2003. Diversity and significance of Burkholderia species occupying diverse ecological niches. Environ. Microbiol. 5:719-729.

14. de Vries, E.J., G. Jacobs, M.W. Sabelis, S.B.J. Menken, and J.A.J. Breeuwer. 2004. Diet-dependent effects of gut bacteria on their insect host: the symbiosis of Erwinia sp. and western flower thrips. Proc. Biol. Sci. 271:2171-2178.

15. Demanèche, S., H. Sanguin, J. Poté, E. Navarro, D. Bernillon, P. Mavingui, W. Wildi, T.M. Vogel, and P. Simonet. 2008. Antibioticresistant soil bacteria in transgenic plant fields. Proc. Natl. Acad. Sci. U.S.A. 105:3957-3962.

16. Elliott, G.N., W.-M. Chen, J.-H. Chou, et al. 2007. Burkholderia phymatum is a highly effective nitrogen-fixing symbiont of Mimosa spp. and fixes nitrogen ex planta. New Phytol. 173:168-80.

17. Ewing, B., and P. Green. 1998. Base-calling of automated sequencer traces using phred. II. Error probabilities. Genome Res. 8:186-194.

18. Ewing, B., L. Hillier, M. Wendl, and P. Green. 1998. Base-calling of automated sequencer traces using phred. I. Accuracy assessment. Genome Res. 8:175-185.

19. Folmer, O., M. Black, W. Hoeh, R. Lutz, and R. Vrijenhoek. 1994. DNA primers for amplification of mitochondrial cytochrome $\mathrm{c}$ oxidase subunit I from diverse metazoan invertebrates. Mol. Mar. Biol. Biotechnol. 3:294-299.

20. Fukatsu, T., and N. Nikoh. 1998. Two intracellular symbiotic bacteria from the mulberry psyllid Anomoneura mori (Insecta: Homoptera). Appl. Environ. Microbiol. 64:3599-3606.

21. Fukatsu, T. 1999. Acetone preservation: a practical technique for molecular analysis. Mol. Ecol. 8:1935-1945.
22. Fukatsu, T., and T. Hosokawa. 2002. Capsule-transmitted gut symbiotic bacterium of the Japanese common plataspid stinkbug, Megacopta punctatissima. Appl. Environ. Microbiol. 68:389-396.

23. Garcia, J.R., A.M. Laughton, Z. Malik, B.J. Parker, C. Trincot, S.S.L. Chiang, E. Chung, and N.M. Gerardo. 2014. Partner associations across sympatric broad-headed bug species and their environmentally acquired bacterial symbionts. Mol. Ecol. 23:1333-1347.

24. Glasgow, H. 1914. The gastric caeca and the caecal bacteria of the Heteroptera. Biol Bull 26:101-170.

25. Henry, T.J. 1997. Phylogenetic analysis of family groups within the infraorder Pentatomomorpha (Hemiptera: Heteroptera), with emphasis on the Lygaeoidea. Ann. Entomol. Soc. Am. 90:275-301.

26. Hosokawa, T., Y. Kikuchi, N. Nikoh, M. Shimada, and T. Fukatsu. 2006. Strict host-symbiont cospeciation and reductive genome evolution in insect gut bacteria. PLoS Biol. 4:e337.

27. Hosokawa, T., N. Kaiwa, Y. Matsuura, Y. Kikuchi, and T. Fukatsu. 2015. Infection prevalence of Sodalis symbionts among stinkbugs. Zool. Lett. 1:5.

28. Hua, J., M. Li, P. Dong, Y. Cui, Q. Xie, and W. Bu. 2008. Comparative and phylogenomic studies on the mitochondrial genomes of Pentatomomorpha (Insecta: Hemiptera: Heteroptera). BMC Genomics 9:610.

29. Inoue, T., O. Kitade, T. Yoshimura, and I. Yamaoka. 2000. Symbiotic associations with protists, p. 275-288. In T. Abe, D.E. Bignell, and M. Higashi (ed.), Termites: Evolution, Sociality, Symbioses, Ecology. Kluwer Academic Publishers, Dordrecht.

30. Ishikawa, T., M. Takai, and T. Yasunaga. 2012. A Field Guide to Japanese Bugs, Vol. 3. Zenkoku Noson Kyoiku Kyokai, Tokyo.

31. Itoh, H., M. Aita, A. Nagayama, X.-Y. Meng, Y. Kamagata, R. Navarro, T. Hori, S. Ohgiya, and Y. Kikuchi. 2014. Evidence of environmental and vertical transmission of Burkholderia symbionts in the oriental chinch bug Cavelerius saccharivorus (Heteroptera: Blissidae). Appl. Environ. Microbiol. 80:5974-5983.

32. Kaiwa, N., T. Hosokawa, Y. Kikuchi, N. Nikoh, X.-Y. Meng, N. Kimura, M. Ito, and T. Fukatsu. 2010. Primary gut symbiont and secondary, Sodalis-allied symbiont of the scutellerid stinkbug Cantao ocellatus. Appl. Environ. Microbiol. 76:3486-3494.

33. Kaiwa, N., T. Hosokawa, N. Nikoh, et al. 2014. Symbiont-supplemented maternal investment underpinning host's ecological adaptation. Curr. Biol. 24:2465-2470.

34. Kaltenpoth, M., S.A. Winter, and A. Kleinhammer. 2009. Localization and transmission route of Coriobacterium glomerans, the endosymbiont of pyrrhocorid bugs. FEMS Microbiol. Ecol. 69:373-383.

35. Katoh, K., and D.M. Standley. 2013. MAFFT multiple sequence alignment software version 7: Improvements in performance and usability. Mol. Biol. Evol. 30:772-780.

36. Kikuchi, Y., X.-Y. Meng, and T. Fukatsu. 2005. Gut symbiotic bacteria of the genus Burkholderia in the broad-headed bugs Riptortus clavatus and Leptocorisa chinensis (Heteroptera: Alydidae). Appl. Environ. Microbiol. 71:4035-4043.

37. Kikuchi, Y., T. Hosokawa, and T. Fukatsu. 2007. Insect-microbe mutualism without vertical transmission: A stinkbug acquires a beneficial gut symbiont from the environment every generation. Appl. Environ. Microbiol. 73:4308-4316.

38. Kikuchi, Y. 2009. Endosymbiotic bacteria in insects: their diversity and culturability. Microbes Environ. 24:195-204.

39. Kikuchi, Y., T. Hosokawa, N. Nikoh, X.-Y. Meng, Y. Kamagata, and T. Fukatsu. 2009. Host-symbiont co-speciation and reductive genome evolution in gut symbiotic bacteria of acanthosomatid stinkbugs. BMC Biol. 7:2.

40. Kikuchi, Y., T. Hosokawa, and T. Fukatsu. 2011. An ancient but promiscuous host-symbiont association between Burkholderia gut symbionts and their heteropteran hosts. ISME J. 5:446-460.

41. Kikuchi, Y., M. Hayatsu, T. Hosokawa, A. Nagayama, K. Tago, and T. Fukatsu. 2012. Symbiont-mediated insecticide resistance. Proc. Natl. Acad. Sci. U.S.A. 109:8618-8622.

42. Kikuchi, Y., T. Hosokawa, N. Nikoh, and T. Fukatsu. 2012. Gut symbiotic bacteria in the cabbage bugs Eurydema rugosa and Eurydema dominulus (Heteroptera: Pentatomidae). Appl. Entomol. Zool. 47:1-8.

43. Kikuchi, Y., and T. Fukatsu. 2014. Live imaging of symbiosis: Spatiotemporal infection dynamics of a GFP-labelled Burkholderia symbiont in the bean bug Riptortus pedestris. Mol. Ecol. 23:14451456. 
44. Kim, J.K., Y.J. Won, N. Nikoh, et al. 2013. Polyester synthesis genes associated with stress resistance are involved in an insect-bacterium symbiosis. Proc. Natl. Acad. Sci. U.S.A. 110:E2381-E2389.

45. Koga, R., T. Tsuchida, and T. Fukatsu. 2009. Quenching autofluorescence of insect tissues for in situ detection of endosymbionts. Appl. Entomol. Zool. 44:281-291.

46. Kuechler, S.M., P. Renz, K. Dettner, and S. Kehl. 2012. Diversity of symbiotic organs and bacterial endosymbionts of: Lygaeoid bugs of the families Blissidae and Lygaeidae (Hemiptera:: Heteroptera: Lygaeoidea). Appl. Environ. Microbiol. 78:2648-2659.

47. Matsuura, Y., Y. Kikuchi, T. Hosokawa, R. Koga, X.-Y. Meng, Y. Kamagata, N. Nikoh, and T. Fukatsu. 2012. Evolution of symbiotic organs and endosymbionts in lygaeid stinkbugs. ISME J. 6:397-409.

48. Matsuura, Y., Y. Kikuchi, X.-Y. Meng, R. Koga, and T. Fukatsu. 2012. Novel clade of alphaproteobacterial endosymbionts associated with stinkbugs and other arthropods. Appl. Environ. Microbiol. 78:4149-4156.

49. Matsuura, Y., T. Hosokawa, M. Serracin, G.M. Tulgetske, T.A. Miller, and T. Fukatsu. 2014. Bacterial symbionts of a devastating coffee plant pest, the stinkbug Antestiopsis thunbergii (Hemiptera: Pentatomidae). Appl. Environ. Microbiol. 80:3769-3775.

50. Miura, T., C. Braendle, A. Shingleton, G. Sisk, S. Kambhampati, and D.L. Stern. 2003. A comparison of parthenogenetic and sexual embryogenesis of the pea aphid Acyrthosiphon pisum (Hemiptera: Aphidoidea). J. Exp. Zool. B Mol. Dev. Evol. 295:59-81.

51. Miyamoto, S. 1961. Comparative morphology of alimentary organs of Heteroptera, with the phylogenetic consideration. Sieboldia 2:197259.

52. Ohbayashi, T., K. Takeshita, W. Kitagawa, et al. 2015. Insect's intestinal organ for symbiont sorting. Proc. Natl. Acad. Sci. U.S.A. 112:E5179-E5188.

53. Olivier-Espejel, S., Z.L. Sabree, K. Noge, and J.X. Becerra. 2011. Gut microbiota in nymph and adults of the giant mesquite bug (Thasus neocalifornicus) (Heteroptera: Coreidae) is dominated by Burkholderia acquired de novo every generation. Environ. Entomol. 40:1102-1110.

54. Onofre-Lemus, J., I, Hernández-Lucas, L. Girard, and J. CaballeroMellado. 2009. ACC (1-aminocyclopropane-1-carboxylate) deaminase activity, a widespread trait in Burkholderia species, and its growthpromoting effect on tomato plants. Appl. Environ. Microbiol. 75: 6581-6590.

55. Prado, S.S., D. Rubinoff, and R.P.P. Almeida. 2006. Vertical transmission of a pentatomid caeca-associated symbiont. Ann. Entomol. Soc. Am. 99:577-585.

56. Prado, S.S., and R.P.P. Almeida. 2009. Phylogenetic placement of pentatomid stink bug gut symbionts. Curr. Microbiol. 58:64-69.

57. Salem, H., E. Kreutzer, S. Sudakaran, and M. Kaltenpoth. 2013. Actinobacteria as essential symbionts in firebugs and cotton stainers (Hemiptera, Pyrrhocoridae). Environ. Microbiol. 15:1956-1968.

58. Salem, H., E. Bauer, A.S. Strauss, H. Vogel, M. Marz, and M. Kaltenpoth. 2014. Vitamin supplementation by gut symbionts ensures metabolic homeostasis in an insect host. Proc. Biol. Sci. 281:20141838.

59. Sawana, A., M. Adeolu, and R.S. Gupta. 2014. Molecular signatures and phylogenomic analysis of the genus Burkholderia: proposal for division of this genus into the emended genus Burkholderia containing pathogenic organisms and a new genus Paraburkholderia gen. nov. harboring environmental species. Front. Genet. 5:1-22.
60. Schaefer, C., and A. Panizzi. 2000. Heteroptera of Economic Importance. CRC Press, Boca Raton, F.L.

61. Schloss, P.D., S.L. Westcott, T. Ryabin, et al. 2009. Introducing mothur: Open-source, platform-independent, community-supported software for describing and comparing microbial communities. Appl. Environ. Microbiol. 75:7537-7541.

62. Schuh, R.T., and J.A. Slater. 1995. True Bugs of the World (Hemiptera: Heteroptera): Classification and Natural History. Cornell University Press, Ithaca.

63. Suárez-Moreno, Z.R., J. Caballero-Mellado, B.G. Coutinho, L. Mendonça-Previato, E.K. James, and V. Venturi. 2012. Common features of environmental and potentially beneficial plant-associated Burkholderia. Microb. Ecol. 63:249-266.

64. Sudakaran, S., H. Salem, C. Kost, and M. Kaltenpoth. 2012. Geographical and ecological stability of the symbiotic mid-gut microbiota in European firebugs, Pyrrhocoris apterus (Hemiptera, Pyrrhocoridae). Mol. Ecol. 21:6134-6151.

65. Sudakaran, S., F. Retz, Y. Kikuchi, C. Kost, and M. Kaltenpoth. 2015. Evolutionary transition in symbiotic syndromes enabled diversification of phytophagous insects on an imbalanced diet. ISME J. 9:25872604.

66. Sun, Y., Z. Cheng, and B.R. Glick. 2009. The presence of a 1-aminocyclopropane-1-carboxylate (ACC) deaminase deletion mutation alters the physiology of the endophytic plant growth-promoting bacterium Burkholderia phytofirmans PsJN. FEMS Microbiol. Lett. 296: $131-136$

67. Tada, A., Y. Kikuchi, T. Hosokawa, D.L. Musolin, K. Fujisaki, and T. Fukatsu. 2011. Obligate association with gut bacterial symbiont in Japanese populations of the southern green stinkbug Nezara viridula (Heteroptera: Pentatomidae). Appl. Entomol. Zool. 46:483-488.

68. Tamura, K., and M. Nei. 1993. Estimation of the number of nucleotide substitutions in the control region of mitochondrial DNA in humans and chimpanzees. Mol. Biol. Evol. 10:512-526.

69. Tamura, K., G. Stecher, D. Peterson, A. Filipski, and S. Kumar. 2013. MEGA6: Molecular evolutionary genetics analysis version 6.0. Mol. Biol. Evol. 30:2725-2729.

70. Vandamme, P., J. Goris, W.-M. Chen, P. de Vos, and A. Willems. 2002. Burkholderia tuberum sp. nov. and Burkholderia phymatum sp. nov., nodulate the roots of tropical legumes. Syst. Appl. Microbiol. 25:507-512.

71. Vandamme, P., K. Opelt, N. Knöchel, C. Berg, S. Schönmann, E. De Brandt, L. Eberl, E. Falsen, and G. Berg. 2007. Burkholderia bryophila sp. nov. and Burkholderia megapolitana sp. nov., moss-associated species with antifungal and plant-growth-promoting properties. Int. J. Syst. Evol. Microbiol. 57:2228-2235.

72. Wang, Q., G.M. Garrity, J.M. Tiedje, and J.R. Cole. 2007. Naïve Bayesian classifier for rapid assignment of rRNA sequences into the new bacterial taxonomy. Appl. Environ. Microbiol. 73:5261-5267.

73. Xie, Q., W. Bu, and L. Zheng. 2005. The Bayesian phylogenetic analysis of the 18S rRNA sequences from the main lineages of Trichophora (Insecta: Heteroptera: Pentatomomorpha). Mol. Phylogenet Evol. 34:448-451. 